\title{
A comparison of 4D time-resolved MRA with keyhole and 3D time-of-flight MRA at 3.0 T for the evaluation of cerebral aneurysms
}

Qian Wu and Ming-Hua Li

\begin{abstract}
Background: A subarachnoid hemorrhage (SAH) due to the rupture of a cerebral aneurysm (CA) is a devastating event associated with high rates of mortality. Magnetic resonance angiography (MRA), as a noninvasive technique, is typically used initially. The object of our study is to evaluate the feasibility of 4D time-resolved MRA with keyhole (4D-TRAK) for the diagnostic accuracy and reliability of the detection and characterization of cerebral aneurysms (CAs), with a comparison of 3D time-of-flight MRA (3D-TOF-MRA) by using DSA as a reference.

Methods: 3D-TOF-MRA, 4D-TRAK and 3D-DSA were performed sequentially in 52 patients with suspected CAs. 4D-TRAK was acquired using a combination of sensitivity encoding (SENSE) and CE timing robust angiography (CENTRA) $\mathrm{k}$-space sampling techniques at a contrast dose of $10 \mathrm{ml}$ at $3 \mathrm{~T}$. Accuracy, sensitivity, specificity of 4D-TRAK and 3D-TOF-MRA were calculated and compared for the detection of CAs on patient-based and aneurysm-based evaluation using 3D-DSA as a reference.
\end{abstract}

Results: The overall image quality of 4D-TRAK with a contrast dose of $10 \mathrm{ml}$ was in the diagnostic range but still cannot be compared with that of 3D-TOF-MRA. In 52 patients with suspected CAs, fifty-eight CAs were confirmed on 3D-DSA finally. Fifty-one (with 2 false-positives and 9 false-negatives) and 58 (with 1 false-positive and 1 false-negative) CAs were visualized on 4D-TRAK and 3D-TOF-MRA, respectively. Accuracy, sensitivity and specificity on patient-based evaluation of 4D-TRAK and 3D-TOF-MRA were $92.31 \%, 93.33 \%, 85.71 \%$ and $98.08 \%, 100 \%, 85.71 \%$, respectively, and $74.07 \%, 75.00 \%, 66.67 \%$ and $96.30 \%, 95.83 \%, 100 \%$ on aneurysm-based evaluation in patients with multiple CAs, respectively. Subgroup analysis revealed that for 19 very small CAs (maximal diameter $<3 \mathrm{~mm}$, measured on 3D-DSA), 9 were missed on 4D-TRAK and 1 on 3D-TOF-MRA $(P=0.008)$. However, for 39 CAs with maximal diameter $\geq 3 \mathrm{~mm}$, the diagnostic accuracy is equally (39 on 4D-TRAK vs. 39 on 3D-TOF-MRA) $(P=1$ ). In four larger CAs with maximal diameter $\geq 10 \mathrm{~mm}$, 4D-TRAK provided a better characterization of morphology than 3D-TOF-MRA.

Conclusion: 4D-TRAK at a lower contrast dose of $10 \mathrm{ml}$ with a combination of SENSE and CENTRA at 3 T could provide similar diagnostic accuracy rate for CAs with maximal diameter $\geq 3 \mathrm{~mm}$, and a better characterization of morphology for larger CAs with maximal diameter $\geq 10 \mathrm{~mm}$ compared to 3D-TOF-MRA. However, further study is still needed to improve the "vascular edge" artifact and the compromise in spatial resolution in depiction of CAs with maximal diameter $<3 \mathrm{~mm}$.

Keywords: 4D time-resolved MRA with keyhole, 3D time-of-flight MRA, DSA, Cerebral aneurysm

\footnotetext{
* Correspondence: liminghuaradio@hotmail.com

Dept of Radiology, The Sixth Affiliated People's Hospital; Medical school of

Shanghai Jiaotong University, No. 600\# Yishan Road, Shanghai 200233, China
} 


\section{Background}

A subarachnoid hemorrhage ( $\mathrm{SAH})$ due to the rupture of a cerebral aneurysm (CA) is a devastating event associated with high rates of mortality. Digital subtraction angiography (DSA) combined with post-processing techniques remains to be the gold standard for detecting and evaluating CAs [1,2]. However, risks include vascular injury, contrast nephrotoxicity, exposure to ionizing radiation and an overall $0.2 \%$ risk of transient and permanent neurological complications [3]. Therefore, as a noninvasive technique, magnetic resonance angiography (MRA) is typically used initially. Although numerous studies have proved that 3D time-of-flight MRA (3D-TOF-MRA) is an effective technique in the detection and characterization of CAs $[4,5]$, disadvantages including long acquisition time, small FOV and flow related artifact remain the problem. The 4D timeresolved MRA with keyhole (4D-TRAK) and a combination of sensitivity encoding (SENSE) and CE timing robust angiography (CENTRA) k-space sampling has demonstrated its wide clinical potential in the characterization of hemodialysis shunts, pulmonary vascular pathologies and cerebral arteriovenous malformations (AVMs) [6-9]. The clinical potential in CAs of this combination of such image acceleration methods includes: 1) better characterization of the morphology of larger CAs better due to its reduced flow related artifact; 2) preoperative assessment of endovascular therapy of CAs by mimicking DSA because of its high spatial and temporal resolution at a larger FOV; 3) fewer motion artifacts as a consequence of the shortened acquisition time for patients with acute aneurysmal SAH. The purpose of our study was to evaluate the feasibility of 4DTRAK for the diagnostic accuracy and reliability of the detection and characterization of CAs, with a comparison of 3D-TOF-MRA by using DSA as a reference and to discuss their respective advantages and disadvantages.

\section{Methods}

\section{Study subjects}

From June 2008 to February 2010, 52 patients with suspected CAs (20 men, 32 women; age range $31-78$ years) were finally enrolled into our study and 6 of them were with acute SAH detected by CT scan. 3D-TOF-MRA, 4D-TRAK and 3D-DSA were performed sequentially to detect the CA occurrence. The study was conducted in accordance with the recommendations of the Declaration of Helsinki, and was approved by the ethics committees in the hospitals. All patients gave written informed consent. Exclusion criteria included age below 18 years, contraindications to MRA examination (e.g. pacemakers, metallic implants), known intolerance to gadolinium contrast agents, with stage 5 CKD (Chronic Kidney Disease, effective glomerular filtration rate $<15 \mathrm{ml} / \mathrm{min} /$
$1.73 \mathrm{~m} \mathrm{[2])} \mathrm{or} \mathrm{on} \mathrm{dialysis,} \mathrm{no} \mathrm{informed} \mathrm{consent} \mathrm{from} \mathrm{pa-}$ tient or qualifying family members, poor neurological condition $(\mathrm{GCS}<12)$, without DSA information acquired contemporaneously or having undergone MRA after a coiling procedure.

\section{Image acquisition \\ MRA}

All MRA examinations were performed on a $3.0 \mathrm{~T}$ system (Achieva X-Series, Philips Healthcare, The Netherlands) with a Sense-Head-8 receiver head coil. 3D-TOF-MRA was conducted using a 3D-T1-FFE sequence with TR/TE, 35/7; flip angle, $20^{\circ}$; field-of-view (FOV), $250 \times 190 \times 108$; four slabs (180 slices); slice thickness, $0.8 \mathrm{~mm}$; matrix, $732 \times 1024$; and acquisition time, 8 min $56 \mathrm{~s}$. We used the 4D-TRAK technique combined with CENTRA k-space sampling and sensitivity encoding (SENSE) to accelerate the image acquisition. 4D-TRAK was acquired using the CENTRA keyhole method with $20 \%$ in the sagittal plane. A central space is randomly filled during the whole passage of the contrast bolus over time and the periphery of $\mathrm{k}$-space was collected in the reference dataset at the end of the acquisition. Sensitivity encoding (SENSE) was used with an acceleration factor (AF) of 4 in the phaseencoding direction and an AF of 2 in the slice-encoding direction yielding a total AF of 8 . The acquisition parameters of the 4D-TRAK technique were as follows: 3DT1-GE sequence with TR/TE, 3.1/1.2; flip angle, $25^{\circ}$; FOV, $230 \times 190 \times 108$; four slabs (273 slices); slice thickness, $0.8 \mathrm{~mm}$; matrix, $201 \times 320$; and acquisition time, $40 \mathrm{~s}$ (reference scanning time, $12 \mathrm{~s} ; 2 \mathrm{~s}$ per phase for 14 phases in total). The 4D-TRAK sequence was started immediately after the start of the injection. A 10-ml bolus of gadopentetate dimeglumine (Gd-DTPA, Magnevist, Bayer-Schering, Germany) was administered at $3 \mathrm{ml} / \mathrm{s}$ and followed by a $20 \mathrm{ml}$ saline injection using a dual-barrel power injection machine at the same rate.

The acquired image data sets were transferred to a workstation (EWS, Philips Medical), where 3D image reconstruction was performed on a $1024 \times 1024$ matrix with volume rendering (VR) and maximum intensity projection (MIP) using a specialist software package (Volume Inspection, Philips Medical).

\section{DSA}

For patients with acute SAH, an interventional neuroradiologist performed DSA within 24 hours after MRA examination, and for those with suspected un-ruptured CAs, DSA was performed within 2 weeks after MRA (between $1 \mathrm{~h}$ and 14 d post-MRA; median, 10.6 days). Conventional 2D-DSA was performed on a monoplanar unit (Axiom Artis VB22N, Siemens Healthcare, Germany) with a $1024 \times 1024$ matrix and $17-20 \mathrm{~cm}$ FOV. Contrast medium was injected for a total of $10 \mathrm{ml}$ for internal 
carotid artery (ICA; $4-5 \mathrm{ml} / \mathrm{s}$ ) and $7 \mathrm{ml}$ for vertebral artery (VA, $2-3 \mathrm{ml} / \mathrm{s}$ ) respectively. Rotational angiography was performed with an 8-s, $200^{\circ}$ rotational run, acquiring 200 images and with the injection of 3-4 ml contrast material per second (total $16-20 \mathrm{ml} /$ artery). 3D images were reconstructed by VR on a workstation with a 128 [3] ×512 [3] matrix (SyngoXWP VA70B, Siemens).

All patients with possible CAs underwent 2D-DSA (including posterior-anterior, lateral and working positions) and 3D-DSA of the affected arteries. 2D-DSA (including posterior-anterior and lateral positions) was performed for the rest of the arteries, and further 3D-DSA would be performed with any positive findings on 2D-DSA. Two experienced observers, who were unaware of all clinical information, identified and evaluated the CAs together.

\section{Image review}

We considered a CA to be a saccular protrusion from the side wall or bifurcation of the cerebral arteries without artery emerging at its top. An infundibulum of maximum diameter $>3 \mathrm{~mm}$ would be considered as a remaining CA and concluded into the statistical analysis in our study. We defined a negative case as a patient with no CA regardless of other cerebrovascular diseases. We ensured that all observers understood these definitions, to achieve consistency of interpretation.

Two experienced observers with no knowledge of the clinical history and DSA results interpreted the rotational 4D-TRAK and 3D-TOF-MRA datasets randomly and independently on an offline workstation, using VR with the single artery highlighting approach from multiple viewing angles. MIPs and source images were presented to the observers with appropriate adjustable thresholds of window width and level. When the two observers could not agree on the presence of a CA, a third observer blind to clinical history and DSA results would be invited and the final decision was reached by a majority.

Image quality of 4D-TRAK and 3D-TOF-MRA was assessed on a four-point scale: 1', inacceptable; 2', acceptable; 3', good; and 4, excellent. The following factors were taken into consideration: background suppression; display of parent artery; display of small terminal branches; venous contamination; and artifacts. Images with a score of 2-4 were considered interpretable. Diagnostic confidence in the presence of a CA on 4D-TRAK and 3D-TOF-MRA was assessed using a previously reported five-point scale: 5 , aneurysm definitely absent; 4 , aneurysm probably absent; 3 , uncertain; 2, aneurysm probably present; and 1, aneurysm definitely present. Cases with one or more aneurysm identified as probably or definitely present were considered positive; all others were considered negative.

\section{Statistical analysis}

The diagnostic performance of 3D-TOF-MRA and 4DTRAK at 3.0 T were compared with that of 3D-DSA. In a patient-based evaluation, a patient with at least one CA detected by both MRA and DSA was considered as a true-positive (TP) case. A patient with no CA detected by MRA and DSA was considered as a true-negative (TF) case. A patient with at least one CA detected by MRA and no CA detected by DSA was considered as a falsepositive (FP) case. A patient with no CA detected by MRA and at least one CA detected by DSA was considered as a false-negative (FN) case. Accuracy, sensitivity, specificity, positive predictive value (PPV) and negative predictive value (NPV) were compared on patient-bypatient level. For patients with multiple CAs, an aneurysm-based evaluation was made as well. Interobserver reliability was calculated for paired observers of 3D-TOF-MRA and 4D-TRAK using the kappa (к) statistic. A $\kappa$ value of 0.8 or above indicated excellent agreement; 0.6-0.8, good agreement; 0.4-0.6, fair agreement; and $<0.4$, poor agreement. The statistical analyses were performed using SPSS version 13.0 (SPSS Inc., Chicago, Illinois).

\section{Results}

Fifty-eight CAs were finally found in 52 patients by 3DDSA, including no CA detected in 7 patients, $1 \mathrm{CA}$ in each of the 34 patients, 2 CAs in each of the 10 patients, and 4 CAs in 1 patient. Nineteen (32.8\%) of the 58 aneurysms were $<3 \mathrm{~mm}$ in maximal diameter, $25(43.1 \%) \geq 3 \mathrm{~mm}$ and $<5 \mathrm{~mm}, 10(17.2 \%) \geq 5 \mathrm{~mm}$ and $<10 \mathrm{~mm}$, and 4 $(6.9 \%) \geq 10 \mathrm{~mm}$. Nine $(15.5 \%)$ aneurysms located at the anterior cerebral artery, $6(10.3 \%)$ at the middle cerebral artery, $35(60.3 \%)$ at the internal carotid artery and $8(13.8 \%)$ at the vertebrobasilar system.

The diagnostic accuracy, sensitivity, specificity, PPV and NPV of 4D-TRAK and 3D-TOF-MRA at $3 \mathrm{~T}$ for the detection of $\mathrm{CA}$ on a patient-based evaluation is described in Table 1. An evaluation based on aneurysmby-aneurysm level for multiple CAs is described in Table 2 and 3. Subgroup analysis revealed that for 19 very small CAs (maximal diameter $<3 \mathrm{~mm}$, measured on 3D-DSA), 9 was missed on 4D-TRAK and 1 on 3DTOF-MRA $(\mathrm{P}=0.008)$. However, for $39 \mathrm{CAs}$ with maximal diameter $\geq 3 \mathrm{~mm}$, the diagnostic accuracy is equally (39 on $4 \mathrm{D}-\mathrm{TRAK}$ vs. 39 on $3 \mathrm{D}$-TOF-MRA) $(\mathrm{P}=1)$. The inter-observer reliability of patient-based evaluation, based on the $\mathrm{k}$ statistic for paired observers, was 0.91 and 0.95 on 4D-TRAK and 3D-TOF-MRA in patientbased evaluation, respectively; 0.89 and 0.96 on 4D- 
Table 1 Diagnostic performance of 4D-TRAK and 3D-TOF-MRA for the detection of CAs identified on 3D-DSA in the patient-based evaluation

\begin{tabular}{lccccccccccc}
\hline Technique & N & TP & TN & FN & k & Sensitivity & Specify\% & Specify\% & PPV\% & NPV\% & Accuracy\% \\
\hline 4D-TRAK & 52 & 42 & 6 & 1 & 3 & 0.91 & 93.33 & 85.71 & 96.67 & 66.67 & 92.31 \\
3D-TOF-MRA & 52 & 45 & 6 & 1 & 0 & 0.95 & 100.00 & 85.71 & 97.83 & 100 & 98.08 \\
\hline
\end{tabular}

Note: $N$ : number; TP: true-positive; TN: true-negative; FP: false-positive; FN: false-negative; $\mathrm{K}$ : kappa statistic; $P P V$ : positive predictive value; $N P V:$ negative predictive value.

TRAK and 3D-TOF-MRA in aneurysm-based evaluation in patients with multiple CAs, respectively.

Summary of misdiagnosed aneurysms on 3D-TOFMRA and 4D-TRAK is detailed in Table 4. In 52 patients, 58 CAs were visualized on 3D-TOF-MRA, 51 on 4D-TRAK (Figure 1). On 3D-TOF-MRA, 1 patient (Case No.41) without any aneurysm was misdiagnosed

Table 2 Diagnostic performance of 4D-TRAK and 3D-TOFMRA for the detection of multiple CAs identified on 3DDSA

\begin{tabular}{|c|c|c|c|c|}
\hline $\begin{array}{l}\text { Case No./ } \\
\text { Gender/Age }\end{array}$ & $\begin{array}{l}\text { Size } \\
(\mathrm{mm})\end{array}$ & Location & 4D-TRAK & 3D-TOF-MRA \\
\hline \multirow[t]{2}{*}{ 10/F/65 } & $\geq 3$ & L C7 segment & $\mathrm{TP}$ & TP \\
\hline & $\geq 3$ & Top of Basilar artery & $\mathrm{TP}$ & TP \\
\hline \multirow[t]{3}{*}{ 16/F/54 } & $<3$ & L C7 segment & FN & TP \\
\hline & $<3$ & L C5 segment & TP & $\mathrm{TP}$ \\
\hline & / & R C5 segment & $\mathrm{TN}$ & $\mathrm{TN}$ \\
\hline \multirow[t]{2}{*}{ 22/M/64 } & $<3$ & L M1 segment & $\mathrm{FN}$ & $\mathrm{TP}$ \\
\hline & $<3$ & L C7 segment & $\mathrm{TP}$ & $\mathrm{TP}$ \\
\hline \multirow[t]{2}{*}{ 26/M/34 } & $\geq 3$ & L C6 segment & $\mathrm{TP}$ & TP \\
\hline & $\geq 3$ & R C5 segment & TP & TP \\
\hline \multirow[t]{5}{*}{$30 / M / 45$} & $<3$ & L P4 segment & FN & $\mathrm{FN}$ \\
\hline & $<3$ & L P2 segment & FN & $\mathrm{TP}$ \\
\hline & $<3$ & L P3 segment & $\mathrm{TP}$ & $\mathrm{TP}$ \\
\hline & / & R C6 segment & TN & TN \\
\hline & $<3$ & R M1 segment & FN & $\mathrm{TP}$ \\
\hline \multirow[t]{3}{*}{ 31/F/59 } & / & L C7 segment & $\mathrm{FP}$ & $\mathrm{TN}$ \\
\hline & $\geq 3$ & R M1 segment & TP & TP \\
\hline & $\geq 3$ & R C7 segment & TP & $\mathrm{TP}$ \\
\hline \multirow[t]{2}{*}{$34 / M / 56$} & $\geq 3$ & R C4 segment & TP & TP \\
\hline & $\geq 3$ & L V4 segment & $\mathrm{TP}$ & $\mathrm{TP}$ \\
\hline \multirow[t]{2}{*}{ 37/F/45 } & $<3$ & L Acom & FN & TP \\
\hline & $\geq 3$ & R C7 segment & $\mathrm{TP}$ & $\mathrm{TP}$ \\
\hline \multirow[t]{2}{*}{ 43/M/40 } & $<3$ & L C6 segment & TP & TP \\
\hline & $<3$ & R Acom & TP & TP \\
\hline \multirow[t]{2}{*}{ 45/M/40 } & $\geq 3$ & L C6 segment & $\mathrm{TP}$ & $\mathrm{TP}$ \\
\hline & $\geq 3$ & R C4 segment & TP & TP \\
\hline \multirow[t]{2}{*}{$52 / M / 46$} & $<3$ & L M1 segment & TP & TP \\
\hline & $\geq 3$ & R Acom & $\mathrm{TP}$ & $\mathrm{TP}$ \\
\hline
\end{tabular}

Note: $M$ : male; F: female; $L$ : left; $R$ : right; Acom: anterior communicating artery; $T P$ : true-positive; $T N$ : true-negative; $F P$ : false-positive; $F N$ : false-negative. due to an acute turn of the siphon at C4. One aneurysm in 1 patient located at left P4 segment (Case No.30) was missed by both the two methods due to its small size and peripheral location. On 4D-TRAK, 2 patients (Case No. 31 and 33) were misdiagnosed as having a aneurysm due to excessively tortuosity at the origin of the posterior communicating artery, and 9 aneurysms were missed in 7 patients (Case No. 3, 16, 21, 22, 27, 30 and 37) with maximal diameter $<3 \mathrm{~mm}$ (Figure 2). In 4 patients with a larger aneurysm $(\geq 10 \mathrm{~mm}$ in diameter), the outline and size can be better ascertained by 4D-TRAK than by 3D-TOF-MRA (Figure 3).

In a semi-quantitative score of image quality of 3DTOF-MRA images, 40 of 52 cases were regarded as excellent (76.0\%), while 10 cases were good (19.2\%) and 2 cases were acceptable (3.8\%). By contrast, the number of excellent cases on 4D-TRAK was only 21 of $52(40.4 \%)$, with 24 good cases (46.2\%) and 7 acceptable cases (13.5\%) $(\mathrm{P}<0.001)$. For 6 restless patients with $\mathrm{SAH}, 4$ cases were acceptable, and 2 cases were good on 3D -TOF-MRA ( 8 min 56 s). On 4D-TRAK (40 s), 2 cases were acceptable, and 4 cases were good.

\section{Discussion}

Time-resolved MRA (TR-MRA) is an alternative MRA technique which uses a keyhole imaging approach to sample the center of $\mathrm{k}$-space more frequently compared with high-spatial-frequency information peripherally to obtain MRA images with high temporal resolution, such as 4D time-resolved MRA with keyhole (4D-TRAK), time-resolved imaging with stochastic trajectories (TWIST), time-resolved echo-shared angiography technique (TREAT), time-resolved imaging of contrast kinetics (TRICKS) [8,10-13]. 4D-TRAK with a combination of parallel imaging and CENTRA at high field strength has demonstrated its wide clinical potential in the characterization of multiple vascular lesions because of the high temporal and spatial resolution obtained simultaneously in previous studies [6-9]. Most ruptured CAs in clinical are $\geq 3 \mathrm{~mm}$ in diameter. In our study, the diagnostic accuracy of TRAK for these CAs is equal to that of 3D-TOF-MRA which has been proved to be of excellent sensitivity, accuracy, and correlation with 3DDSA, as it was demonstrated in the previous studies in a large cohort of patients in our center $[14,15]$. For uncooperative patients with acute SAH and renal impairment 
Table 3 Diagnostic performance of 4D-TRAK and 3D-TOF-MRA for the detection of CAs identified on 3D-DSA in the aneurysm-based evaluation in patients with multiple CAs

\begin{tabular}{lccccccccccc}
\hline Technique & N & TP & TN & FN & k & Sensitivity & Specify\% & Specify\% & PPV\% & NPV\% & Accuracy\% \\
\hline 4D-TRAK & 19 & 18 & 2 & 1 & 6 & 0.89 & 75.00 & 66.67 & 94.74 & 25.00 & 74.07 \\
3D-TOF-MRA & 23 & 23 & 3 & 0 & 1 & 0.96 & 95.83 & 100.00 & 100.00 & 75.00 & 96.30 \\
\hline
\end{tabular}

Note: $N$ : number; TP: true-positive; TN: true-negative; FP: false-positive; FN: false-negative; k: kappa statistic; PPV: positive predictive value; NPV: negative predictive value.

at the same time, both computed tomographic angiography (CTA) and 3D-TOF-MRA could not be considered as the first option because of the renal impairment associated with the iodinated contrast agents and the possibility of more motion artifacts due to a relatively longer acquisition time (8 $\mathrm{min} 56 \mathrm{~s}$ in our study), respectively. Considering the increasing current concern linking high-dose gadolinium $(\mathrm{Gd})$ chelates to NSF in patients with advanced renal failure [16,17], a MRA protocol with low-dose contrast medium was desired for CAs patients with renal impairment. However, the desirable dose is frequently too small to perform both a timing bolus and non time-resolved contrast-enhanced MRA (CE-MRA). Although it was reported that the lowdose contrast medium with the associated shorter duration of bolus may have contributed to a compromise of signal-to-noise ratio (SNR) and contrast-to-noise ratio (CNR) and lead to a suboptimal small-vessel conspicuity [18], benefiting from higher magnetic fields with an associated gain in SNR as well as effects on T1relaxation of contrast $[19,20]$ and subtraction imaging for background suppression and contrast enhancement [21], the small dose of contrast agent utilized has proved to be sufficient to provide dynamic, functional, as well as morphological information in a wide range of vascular diseases in TR-MRA [22,23]. In our result, 4D-TRAK with a single injection of $10 \mathrm{ml}$ of Gd-DPTA and a relatively shorter acquisition time of 40s was sufficient to provide a frame rate up to 2 seconds and morphological information as well and could become a supplementary method for some uncooperative patients with acute $\mathrm{SAH}$ and with renal inadequacy.

However, for small CAs with maximal diameter $<3 \mathrm{~mm}$, it does not yet have a diagnostic accuracy comparable with 3D-TOF-MRA. The "vascular edge" artifact (blurring or irregular enhancement of vascular edges) and a compromise of spatial resolution compared with high resolution imaging such as 3D-TOF-MRA may be a diagnostic limitation for the overall image quality of 4DTRAK and confident evaluation of smaller CAs. The peripheral parts of k-space, necessary for spatial resolution, are acquired with the keyhole reference scan after all dynamics in CENTRA keyhole. The sequence that combined CENTRA keyhole and SENSE in our study was likely to provide images with an increased background noise level and reduced vessel delineation due to absence of gadolinium in wash-out phase compared with CE-MRA. A CA is defined as a saccular protrusion from the side wall or bifurcation of the cerebral arteries without artery emerging at its top. Irregular enhancement of vascular edges would reduce the slope-rates of protrusion on vessel edge, as a result of decreasing the

Table 4 Summary of false-positive and false-negative aneurysms on 4D-TRAK and 3D-TOF-MRA

\begin{tabular}{|c|c|c|c|c|c|}
\hline Case No./Gender/Age & 4D-TRAK & 3D-TOF-MRA & Location & Size $(\mathrm{mm})$ & Causes \\
\hline $31 / F / 59$ & $\mathrm{FP}$ & $\mathrm{TN}$ & L C7 segment & $\geq 3$ & \multirow[t]{2}{*}{ Excessively tortuosity at the origin of the Pcom } \\
\hline $33 / F / 58$ & $\mathrm{FP}$ & $\mathrm{TN}$ & L C7 segment & $\geq 3$ & \\
\hline $30 / M / 40$ & FN & FN & L P4 segment & $<3$ & Distal location and small size \\
\hline $16 / F / 54$ & FN & TP & L C7 segment & $<3$ & \multirow{8}{*}{$\begin{array}{l}\text { "vascular edge" artifact and spatial resolution } \\
\text { compromise of 4D-TRAK technique }\end{array}$} \\
\hline $37 / F / 45$ & FN & TP & L Acom & $<3$ & \\
\hline 22/M/64 & FN & TP & L M1 segment & $<3$ & \\
\hline $30 / M / 45$ & FN & TP & LP2 segment & $<3$ & \\
\hline $21 / \mathrm{M} / 60$ & FN & $\mathrm{TP}$ & R C4 segment & $<3$ & \\
\hline 3/M/35 & FN & TP & L C5 segment & $<3$ & \\
\hline 27/M/45 & FN & $\mathrm{TP}$ & R C5 segment & $<3$ & \\
\hline $30 / M / 45$ & FN & TP & R M1 segment & $<3$ & \\
\hline $41 / M / 58$ & TN & $\mathrm{FP}$ & L C4 segment & $\geq 3$ & Acute turn of the siphon at $\mathrm{C} 4$ segment \\
\hline
\end{tabular}

Note: M: male; F: female; L: left; $R$ : right; Acom: anterior communicating artery; Pcom: posterior communicating artery; TP: true-positive; TN: true-negative; FP: falsepositive; $F N$ : false-negative. 


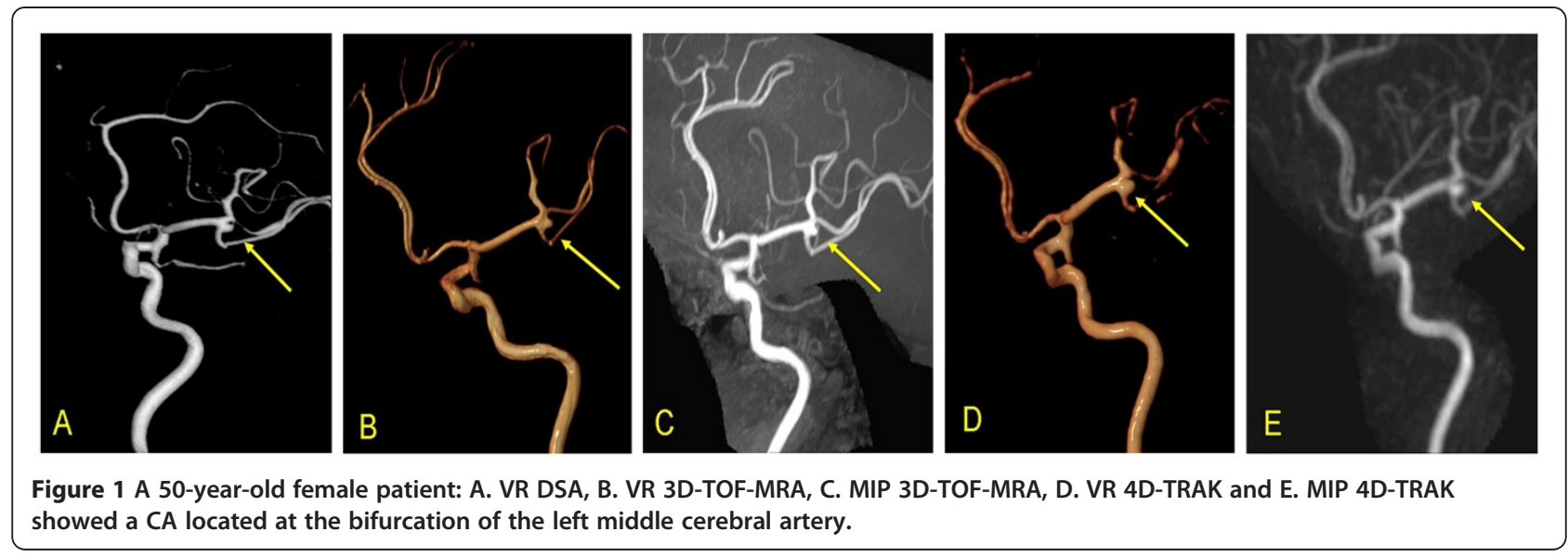

diagnostic tendency of CAs or misdiagnosing as unsmooth arterial walls due to atherosclerosis, especially for CAs with a smaller size.

4D-TRAK could also be potential in depiction the morphology of larger CAs in patients with renal insufficiency. Compared with 3D-TOF-MRA, the decreased sensitivity to saturation and turbulence effect can be the advantage of 4D-TRAK benefiting from its different imaging principle $[24,25]$. Within larger aneurysms, especially those with partial thrombosis, complex flow is usual found [26]. Similar to Jäge's results [27], we found 4D-TRAK to characterize the morphology of lesions better than 3D-TOF-MRA compared to 3D-DSA in four larger aneurysms $(\geq 10 \mathrm{~mm})$. However, the diagnostic sensitivity of 3D-TOF-MRA was not decreased in these cases.

After referencing the previous studies [28-31], except the decreasing risk of NSF for patients with renal failure, advantages of 4D-TRAK compared with non timeresolved CE-MRA in the detection of CAs include: 1) no need for individual assessment of bolus arrival time; Timing of the Gd bolus relative to acquisition of central k-space is critical for CE- MRA for maximum arterial signal with minimal venous signal $[28,29]$. It can be performed by a separate timing bolus injection or by bolustracking sequences that allow automatic or manual triggering when contrast material is seen in the vessels to be imaged. Compared with CE MRA,no additional bolus timing and time-consuming sequence adjustments are necessary in time-resolved imaging. One simply injects the contrast material and performs the scan simultaneously, because as a result of the high temporal update rate ( $2 \mathrm{~s}$ in our study), one of the volumes will coincide with the arrival of contrast material. For CAs patients accompany with any of the following: pediatric patients with fast contrast media passage, patients with impaired cardiac output, and patients with high dilution volumes, the facilitated application of contrast media without the need for an optimal bolus timing may be especially helpful [30]. 2) less arterial and venous overlap; Although CE-MRA has a high spatial resolution for greater anatomical definition of vessels, there is also an increased chance for arterial and venous overlap, which can limit the diagnostic accuracy of CAs [31]. 3) to have the

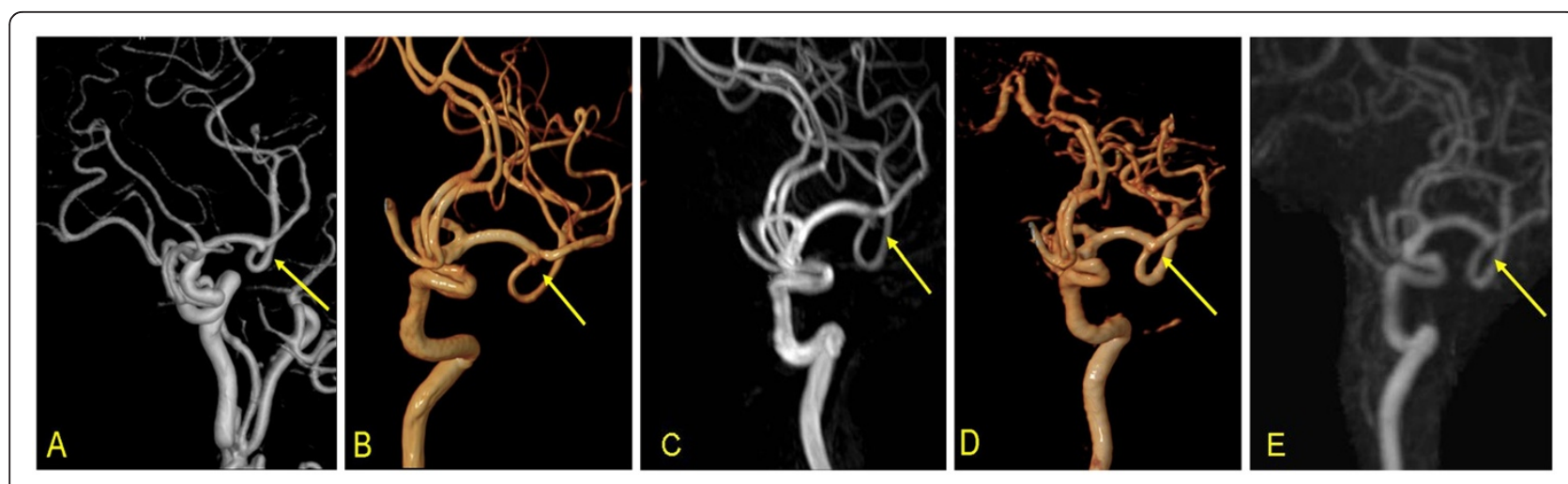

Figure 2 A 45-year-old male patient: A. VR DSA, B. VR 3D-TOF-MRA and C. MIP 3D-TOF-MRA showed a CA located at the bifurcation of left middle cerebral artery. D. VR 4D-TRAK and E. MIP 4D-TRAK missed the aneurysm. 


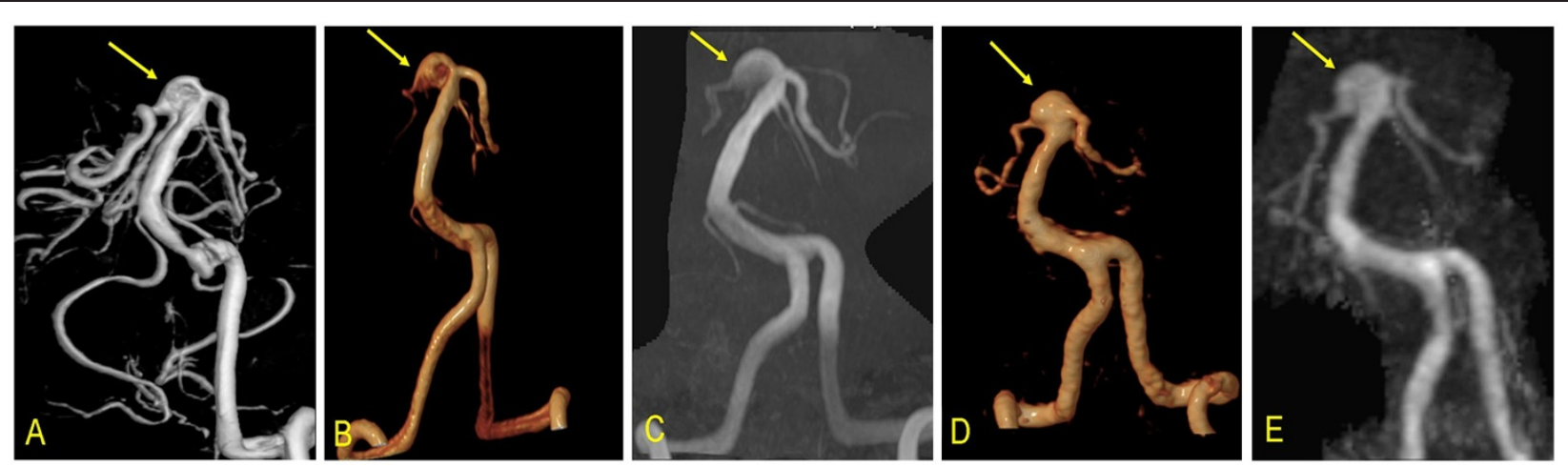

Figure 3 A 65-year-old female patient: A. VR DSA, D. VR 4D-TRAK and E. MIP 4D-TRAK have better characterization of the CA (11 mm in maximum diameter) located at the top of the basal artery than B.VR 3D-TOF-MRA and C. MIP 3D-TOF-MRA.

potential to help the decision-making process of endovascular strategy for CAs because of the ability to follow the course of blood flow with an angiographic overview similar to that of DSA. However, further study is still needed to obtain the higher spatial and temporal resolution for a better mimic of process of angiography.

We acknowledge the following limitations of our study. 1) Our study didn't obtain conventional non-time resolved CE-MRA sequences at the same time, allowing for avoiding the second contrast injection. 2) Our data were obtained from a relatively small sample size.

\section{Conclusion}

4D-TRAK at a lower contrast dose of $10 \mathrm{ml}$ with a combination of SENSE and CENTRA at $3 \mathrm{~T}$ could provide similar diagnostic accuracy rate for CAs with maximal diameter $\geq 3 \mathrm{~mm}$, and a better characterization of morphology for larger CAs with maximal diameter $\geq 10 \mathrm{~mm}$ compared to 3D-TOF-MRA. However, further study is still needed to improve the "vascular edge" artifact and the compromise in spatial resolution in depiction of CAs with maximal diameter $<3 \mathrm{~mm}$.

\section{Competing interests}

Both authors declare that they have no competing interests.

\section{Authors' contributions}

WQ and LMH both carried out the study design, patients selection and inclusion, image analysis, statistical analysis and drafted the manuscript. WQ and LMH made the revision and approved the final manuscript. All authors read and approved the final manuscript.

Received: 26 January 2012 Accepted: 15 June 2012

Published: 6 July 2012

\section{References}

1. Zhou B, Li MH, Wang W, Xu HW, Cheng YD, Wang J: Three-dimensional volume-rendering technique in the angiographic follow-up of cerebral aneurysms embolized with coils. J Neurosurg 2010, 112(3):674-680.

2. Shi WY, Li YD, Li MH, Gu BX, Chen SW, Wang W, Zhang BL, Li M: 3D rotational angiography with volume rendering: The utility in the detection of cerebral aneurysms. Neurol India 2010, 58(6):908-913.
3. Kaufmann TJ, Huston J 3rd, Mandrekar JN, Schleck CD, Thielen KR, Kallmes DF: Complications of diagnostic cerebral angiography: evaluation of 19,826 consecutive patients. Radiology 2007, 243(3):812-819.

4. Tang PH, Hui F, Sitoh YY: Cerebral aneurysm detection with $3 \mathrm{~T}$ magnetic resonance angiography. Ann Acad Med Singapore 2007, 36(6):388-393.

5. White PM, Wardlaw JM, Easton V: Can noninvasive imaging accurately depict intracranial aneurysms? A systematic review. Radiology 2000, 217(2):361-370.

6. Mende KA, Froehlich JM, von Weymarn C, Hoogeveen R, Kistler T, Zollikofer $\mathrm{CL}$, Wentz KU: Time-resolved, high-resolution contrast-enhancaed MR angiography of dialysis shunts using the CENTRA keyhole technique with parallel imaging. J Magn Reson Imaging 2007, 25(4):832-840.

7. Taschner CA, Gieseke J, Le Thuc V, Rachdi H, Reyns N, Gauvrit JY, Leclerc X Cerebral arteriovenous malformation: time-resolved contrast-enhanced MR angiography with combination of parallel imaging, keyhole acquisition, and k-space sampling techniques at 1.5 T. Radiology 2008, 246(3):871-879.

8. Willinek WA, Hadizadeh DR, von Falkenhausen M, Urbach $H$, Hoogeveen $R$, Schild HH, Gieseke J: 4D time-resolved MR angiography with keyhole (4D-TRAK): more than 60 times accelerated MRA using a combination of CENTRA, keyhole, and SENSE at 3.0 T. J Magn Reson Imaging 2008, 27(6):1455-1460

9. Körperich H, Gieseke J, Esdorn H, Peterschröder A, Hoogeveen R, Barth P, Peuster M, Meyer H, Sarikouch S, Beerbaum P: Ultrafast time-resolved contrast-enhanced 3D pulmonary venous cardiovascular magnetic resonance angiography using SENSE combined with CENTRA-keyhole. J Cardiovasc Magn Reson 2007, 9(1):77-87.

10. Lima RP, Shapiroa M, Wanga EY, Lawc M, Babba JS, Rueffa LE, Jacoba JS, Kima S, Carsona RH: C, T.P. Mulhollanda, G. Laubb and E.M. Hechta. 3D Time-Resolved MR Angiography (MRA) of the Carotid Arteries with Time-Resolved Imaging with Stochastic Trajectories: Comparison with 3D Contrast-Enhanced Bolus-Chase MRA and 3D Time-Of-Flight MRA. AJNR Am J Neuroradiol 2008, 29(10):1847-1854.

11. Fink C, Ley S, Kroeker R, Requardt M, Kauczor HU: Bock MTime-resolved contrast-enhanced three-dimensional magnetic resonance angiography of the chest: combination of parallel imaging with view sharing (TREAT). Invest Radiol 2005, 40:40-48.

12. Riederer SJ, Tasciyan T, Farzaneh F, Lee JN, Wright RC, Herfkens RJ: MR fluoroscopy: technical feasibility. Magn Reson Med 1988, 8:1-15.

13. Fink C, Puderbach M, Ley S, Zaporozhan J, Plathow C, Kauczor HU: Time-resolved echo-shared parallel MRA of the lung: observer preference study of image quality in comparison with non-echo-shared sequences. Eur Radiol 2005, 15:2070-2074.

14. Li MH, Li YD, Tan HQ, Gu BX, Chen YC, Wang W, Chen SW: HuDJ Contrastfree MRA at 3.0 T for the detection of intracranial aneurysms. Neurology 2011, 77(7):667-676.

15. Ming-Hua L, Hao-Wen Xu: Large-Cohort Comparison Between ThreeDimensional Time-of-Flight Magnetic Resonance and Rotational Digital Subtraction Angiographies in Intracranial Aneurysm Detection. Stroke 2009, 40:3127-3129. 
16. Hedley AJ, Molan MP, Hare DL, et al: Nephrogenic systemic fibrosis associated with gadolinium-containing contrast media administration in patients with reduced glomerular filtration rate. Nephrology (Carlton) 007 2011, 12(1):111. Feb.

17. Sadowski EA, Bennett LK, Chan MR, et al: Nephrogenic systemic fibrosis: risk factors and incidence estimation. Radiology 2007, 243(1):148-157.

18. Frayne R, Grist TM, Swan JS, et al: 3D MR DSA: effects of injection protocol and image masking. J Magn Reson Imaging 2000, 12:476-487.

19. Norris DG: High field human imaging. J Magn Reson Imaging 2003, 18:519-529.

20. Lotz J, Doker R, Noeske R, Schuttert M, Felix R, Galanski M, Gutberlet M, Meyer GP: In vitro validation of phase-contrast flow measurements at $3 \mathrm{~T}$ in comparison to $1.5 \mathrm{~T}$ : precision, accuracy, and signal-to-noise ratios. J Magn Reson Imaging 2005, 21:604-610.

21. Grobner T: Gadolinium: a specific trigger for the development of nephrogenic fibrosing dermopathy and nephrogenic systemic fibrosis? Nephrol Dial Transplant 2006, 21:1104-1108.

22. Krishnam MS, Tomasiana A, Lohana DG, Trana L, Finna JP, Ruehma SG: Lowdose, time-resolved, contrast-enhanced 3D MR angiography in cardiac and vascular diseases: correlation to high spatial resolution $3 \mathrm{D}$ contrast-enhanced MRA. Clin Radiol 2008, 63(7):744-755.

23. Lee Y-J, Laub G, Jung So-Lyung, Yoo W-J, Kim Y-J, Ahn K-J, Kim Bum-soo: Low-Dose 3D Time-Resolved Magnetic Resonance Angiography (MRA) of the Supraaortic Arteries: Correlation With High Spatial Resolution 3D Contrast-Enhanced MRA. J Magn Reson Imaging 2011, 33:71-76.

24. Parker DL, Tsuruda JS, Goodrich KC, Alexander AL, Buswell HR: Contrastenhanced magnetic resonance angiography of cerebral arteries. A review. Invest Radiol 1998, 33(9):560-572.

25. Isoda H, Takehara Y, Isogai S, Masunaga H, Takeda H, Nozaki A, Sakahara H: MRA of cerebral aneurysm models: a comparison of contrast-enhanced three-dimensional MRA with time-of-flight MRA. J Comput Assist Tomogr 2000, 24(2):308-315.

26. Brugières $P$, Blustajn J, Le Guérinel C, Méder JF, Thomas P, Gaston A: Magnetic resonance angiography of giant cerebral aneurysms. Neuroradiology 1998, 40(2):96-102.

27. Jäger HR, Ellamushi H, Moore EA, Grieve JP, Kitchen ND, Taylor WJ Contrast-enhanced MR angiography of cerebral giant aneurysms. Am J Neuroradiol 2000, 21:1900-1907.

28. Sabati M, Lauzon ML, Frayne R: Space-time relationship in continuously moving table method for large FOV peripheral contrastenhanced magnetic resonance angiography. Phys Med Biol 2003, 7:17.

29. Maki JH, Prince MR, Londy FJ, Chenevert TL: The effects of time varying intravascular signal intensity and k-space acquisition order on threedimensional MR angiography image quality. J Magn Reson Imaging 1996, 6:642-651.

30. Alex Frydrychowicz, Bley Thorsten A, Jan T, WintererAndreas Harloff, Mathias Langer, Jürgen Hennig, Michael Markl: Accelerated time-resolved 3D contrast-enhanced MR angiography at $3 \mathrm{~T}$ : clinical experience in 31 patients. Magn Reson Mater Phy 2006, 19:187-195.

31. Baskaran V, Pereles FS, Nemcek A, et al: Gadolinium-enhanced 3D MR angiography of renal artery stenosis: a pilot comparison of maximumintensity-projection, multiplanar reformatting and 3D volume rendering post-processing algorithms. Acad Radiol 2002, 9:50-59.

doi:10.1186/1471-2377-12-50

Cite this article as: Wu and Li: A comparison of $4 \mathrm{D}$ time-resolved MRA with keyhole and 3D time-of-flight MRA at 3.0 T for the evaluation of cerebral aneurysms. BMC Neurology 2012 12:50.

\section{Submit your next manuscript to BioMed Central and take full advantage of:}

- Convenient online submission

- Thorough peer review

- No space constraints or color figure charges

- Immediate publication on acceptance

- Inclusion in PubMed, CAS, Scopus and Google Scholar

- Research which is freely available for redistribution

Submit your manuscript at www.biomedcentral.com/submit
Ciomed Central 